\title{
Qubit-induced phonon blockade as a signature of quantum behavior in nanomechanical resonators
}

\author{
Yu-xi Liu, ${ }^{1,2,3}$ Adam Miranowicz, ${ }^{1,4}$ Y. B. Gao, ${ }^{5}$ Jiří Bajer, ${ }^{6}$ C. P. Sun, ${ }^{7}$ and Franco Nori ${ }^{1,8}$ \\ ${ }^{1}$ Advanced Science Institute, RIKEN, Wako-shi, Saitama 351-0198, Japan \\ ${ }^{2}$ Institute of Microelectronics, Tsinghua University, Beijing 100084, China \\ ${ }^{3}$ Tsinghua National Laboratory for Information Science and Technology (TNList), Tsinghua University, Beijing 100084, China \\ ${ }^{4}$ Faculty of Physics, Adam Mickiewicz University, 61-614 Poznań, Poland \\ ${ }^{5}$ College of Applied Science, Beijing University of Technology, Beijing, 100124, China \\ ${ }^{6}$ Department of Optics, Palacký University, 77200 Olomouc, Czech Republic \\ ${ }^{7}$ Institute of Theoretical Physics, The Chinese Academy of Sciences, Beijing, 100080, China \\ ${ }^{8}$ Physics Department, The University of Michigan, Ann Arbor, Michigan 48109-1040, USA
}

(Dated: October 8, 2018)

\begin{abstract}
The observation of quantized nanomechanical oscillations by detecting femtometer-scale displacements is a significant challenge for experimentalists. We propose that phonon blockade can serve as a signature of quantum behavior in nanomechanical resonators. In analogy to photon blockade and Coulomb blockade for electrons, the main idea for phonon blockade is that the second phonon cannot be excited when there is one phonon in the nonlinear oscillator. To realize phonon blockade, a superconducting quantum two-level system is coupled to the nanomechanical resonator and is used to induce the phonon self-interaction. Using Monte Carlo simulations, the dynamics of the induced nonlinear oscillator is studied via the Cahill-Glauber $s$-parametrized quasiprobability distributions. We show how the oscillation of the resonator can occur in the quantum regime and demonstrate how the phonon blockade can be observed with currently accessible experimental parameters.
\end{abstract}

PACS numbers: 85.85.+j, 03.65.Yz, 85.25.Cp, 42.50.Dv

\section{INTRODUCTION}

Many efforts (e.g., see Refs. [1-4] and reviews [5-7]) have been made to explore quantum effects in nanomechanical resonators (NAMRs) and optomechanical systems (e.g., in Refs. [8-13] and the review [14]). Reaching the quantum limit of NAMRs would have important applications in, e.g., small mass or weak-force detections [15-17], quantum measurements [18], and quantum-information processing. Only recently the quantum limit in NAMRs has been reached experimentally [19].

Quantum or classical behavior of a NAMR oscillation depends on its environment, which induces the decoherence and dissipation of the NAMR states. In principle, if the NAMR is cooled to very low temperatures (in the mK-range) and has sufficiently high oscillation frequencies (in the GHz-range), then its oscillation can approach the quantum limit. In other words, if the energies of the NAMR quanta, which are referred to as phonons [20], are larger than (or at least comparable to) the thermal energy, then the mechanical oscillation can be regarded quantum. When the NAMR can beat the thermal energy and approach the quantum regime, measurements on quantum oscillation of the NAMRs are still very challenging. One encounters: (i) fundamental problems as measurements are usually performed by the position detection, the quantum uncertainty due to the zero-point fluctuation will limit the measurement accuracy; (ii) practical problems as, for a beam oscillating with frequency in the gigahertz range, the typical displacement for this oscillation is on the order of a femtometer. Detecting so tiny displacement is a difficult task for current experimental techniques.

Various signatures and applications of quantum behavior (or nonclassicality) in nanomechanical resonators have been studied. Examples include: generation of quantum entangle- ment [21-23], generation of squeezed states [24-27], Fock states [28, 29], Schrödinger cat states [30], and other nonclassical states [31, 32], prediction of classical-like [33] and quantum [34] Rabi oscillations, transport measurements [35], quantum nondemolition measurements [18, 28, 29, 36, 37], quantum tunneling [38], proposal of quantum metrology [39] and of quantum decoherence engineering [25].

The problem of how to perform quantum measurements on a system containing a NAMR plays a fundamental role in reaching the quantum limit of the NAMR and testing its nonclassical behavior. Quantum measurements are usually done by coupling an external probe (detector) to the NAMR (see, e.g., [2, 4, 32, 40-44] and references therein).

Our approach for detecting quantum oscillations of NAMRs is based on: (i) recent theoretical proposals (e.g., Refs. [45]) to perform quantum measurement on NAMR without using an external probe and (ii) experimental demonstrations (e.g., Refs. [46, 47]) on the couplings between superconducting quantum devices and the NAMRs. Instead of directly detecting a tiny displacement, we propose to indirectly observe quantum oscillations of the NAMR via phonon blockade, which is a purely quantum phenomenon.

We assume that the phonon decay rate is much smaller than the phonon self-interaction strength. In such a case, we show that when the oscillations of the NAMR are in the nonclassical regime, the phonon excitation can be blockaded. In analogy to the photon (e.g., see Refs. [48, 49]) and Coulomb (e.g., see Ref. [50]) blockades, the main idea for the phonon blockade is that the second phonon cannot be excited when there is one phonon in the nonlinear oscillator. Therefore, by analyzing correlation spectra for the electromotive force generated between two ends of the NAMR, the phonon blockade can be distinguished from excitations of two or more phonons.

An important ingredient for the realization of the phonon 


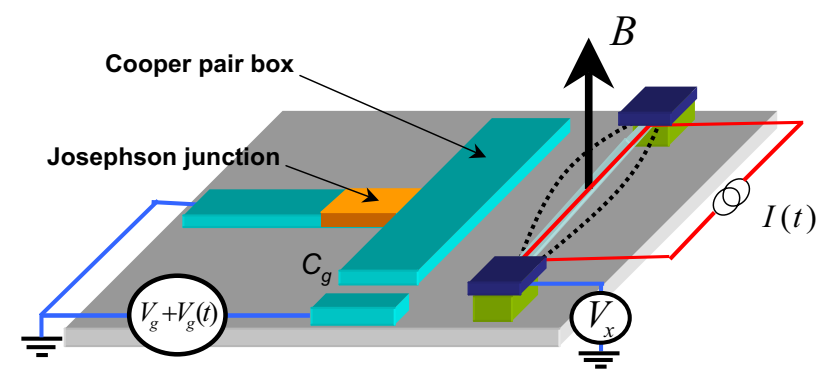

FIG. 1: (Color online) Schematic diagram for the coupling between a nanomechanical resonator (light blue bar on the right side) and superconducting charge qubit (left side). Two black dashed curves on the right show that the resonator is oscillating. The static magnetic field $B$ (presented by the black upward-pointing arrow) and the alternating current $I(t)$ (shown by the red loop on the right) are used for the motion detection of the NAMR.

blockade is strong phonon self-interaction. To obtain such nonlinear phonon-phonon interaction, the NAMR is assumed to be coupled to a superconducting two-level system, which can be either charge, flux, or phase qubit circuits [51-54]. By choosing appropriate parameters of two-level systems, a nonlinear phonon interaction can be induced. The interactions between each of these qubits [51-54] with NAMRs are very similar, e.g., the coupling constants are of the same order and the frequencies of these qubits are in the same $\mathrm{GHz}$ range. Therefore, in this paper, we only use charge qubits as an example to demonstrate our approach. However, this approach can also be applied to demonstrate the oscillation of the NAMR in the quantum regime, when the NAMR is coupled to other superconducting qubits, e.g., phase or flux qubits.

The paper is organized as follows. In Sec. II, we describe the couplings between the superconducting qubits and the NAMR, and then study how the qubit induces the phononphonon interaction. In Sec. III, we discuss how to characterize the quantum oscillation by using the Cahill-Glauber $s$ parametrized quasiprobability distributions for $s>0$, in contrast to the Wigner function (for $s=0$ ). In Sec. IV, the basic principle of the phonon blockade is demonstrated, and we show that the phonon blockade can occur for the different parameters. In Sec. V, we study the measurement of the phonon blockade by using the correlation spectrum of the electromotive force between two ends of the NAMR. Finally, we summary the main results of the paper in Sec. VI.

\section{QUBIT-INDUCED PHONON-PHONON INTERACTION}

Let us now focus on the coupling between a NAMR (with mass $m$ and length $L$ ) and a superconducting charge qubit (with Josephson energy $E_{J}$ and junction capacitance $C_{J}$ ). As schematically shown in Fig. 1 a direct-current (d.c.) voltage $V_{g}$, and an a.c. voltage $V_{g}(t)=V_{0} \cos \left(\omega_{1} t\right)$ are applied to the charge qubit (or Cooper pair box) through the gate capacitor $C_{g}$. The NAMR is coupled to the charge qubit by applying a static voltage $V_{x}$ through the capacitor $C(x)$ which depends on the displacement $x$ of the NAMR around its equilibrium position. A weak detecting current $I(t)=I_{0} \cos \left(\omega_{2} t\right)$ is applied to the NAMR, with its long axis perpendicular to the static magnetic field $B$.

In the rotating wave approximation and neglecting twophonon terms, the Hamiltonian $H=H_{0}+H_{\mathrm{d}}$ of the interaction system between the charge qubit and the NAMR can be described by [45]:

$$
\begin{aligned}
H^{(0)}= & \frac{1}{2} \hbar \omega_{0} \sigma_{z}+\hbar \omega a^{\dagger} a+\hbar g\left(a \sigma_{+}+a^{\dagger} \sigma_{-}\right) \\
& +\hbar \Omega\left(\sigma_{+} e^{-i \omega_{1} t}+\sigma_{-} e^{i \omega_{1} t}\right), \\
H^{(\mathrm{d})}= & \hbar \epsilon\left(a^{\dagger} e^{-i \omega_{2} t}+a e^{i \omega_{2} t}\right) .
\end{aligned}
$$

Here, the frequency shift of the NAMR, due to its coupling to the charge qubit, has been neglected because it just renormalizes the NAMR frequency and will not affect the calculations below. This frequency shift is determined [31] by the qubitNAMR distance $l$, the charging energy $E_{c}=e^{2} / 2\left(C_{J}+C_{g}+\right.$ $C)$, the mass $m$ and the frequency $\omega$ of the NAMR. It should be noted that below we consider the large detuning between the qubit and the NAMR, i.e., $\left(\omega_{0}-\omega\right)$ is several times larger (but not much larger) than the coupling constant $g$; thus, the rotating wave approximation can be applied. The effect of the counter-rotating terms on the results can also calculated in the large detuning case [55]. However, here we have neglected this effect because it only produces a small frequency shift and two-photon processes. The charge qubit, described by the spin operator $\sigma_{z}=|e\rangle\langle e|-| g\rangle\langle g|$, is assumed to be near the optimal point, i.e., $\left(C_{g} V_{g}+C V_{x}\right) / 2 e \approx 0.5$ with $C=C(x=0)$, and thus $\omega_{0} \approx E_{J} / \hbar$. The qubit ground and excited states are denoted by $|g\rangle$ and $|e\rangle$, respectively. The operator $a\left(a^{\dagger}\right)$ denotes the annihilation (creation) operator of the NAMR with frequency $\omega$, which can be written as

$$
\begin{aligned}
a & =\sqrt{\frac{m \omega}{2 \hbar}}\left(x+\frac{i}{m \omega} p\right), \\
a^{\dagger} & =\sqrt{\frac{m \omega}{2 \hbar}}\left(x-\frac{i}{m \omega} p\right)
\end{aligned}
$$

with the momentum operator $p$ of the NAMR. The third term of Eq. (1) presents the NAMR-qubit interaction with the strength

$$
g=\frac{4 E_{c} N_{x} X_{0}}{d}
$$

determined by the charging energy $E_{c}$, effective Copper pair number $N_{x}=C V_{x} / 2 e$, the distance $d$ between the NAMR and the superconducting qubit, and the NAMR amplitude $X_{0}=\sqrt{\hbar / 2 m \omega}$ of zero-point motion. Also, $\Omega$ is the Rabi frequency of the qubit driven by the classical field with frequency $\omega_{1}$. The parameter

$$
\epsilon=-B I_{0} L X_{0}
$$

in Eq. (2) describes the interaction strength between the NAMR and an external weak probe a.c. current with frequency $\omega_{2}$. Hereafter, we assume that the resonant driving 
condition for the qubit is satisfied, i.e., $\omega_{1}=\omega_{0}$. For the coupling between a phase [21] (or flux qubit [46]) and the NAMR, they also have the same form as that given in Eqs. (1) and (2) except all parameters of the Hamiltonian should be specified to the concrete systems. Thus, our discussions below can also be applied to those systems.

The frequency of the NAMR is usually much lower than that of the qubit. If the Rabi frequency $\Omega$ satisfies the condition $\Omega \gg\left(g^{2} / \Delta\right)$ with the detuning $\Delta=\omega_{0}-\omega$ between the frequencies of the NAMR and the qubit, then in the rotating reference frame with $V=\exp \left(-i \omega_{0} \sigma_{z} / 2\right)$, Eq. (1) is equivalent to an effective Hamiltonian

$$
H_{\mathrm{eff}}^{(0)}=\hbar \omega a^{\dagger} a+\hbar\left[\frac{g^{2}}{\Delta} a^{\dagger} a-\kappa\left(a^{\dagger} a\right)^{2}\right] \rho_{z}
$$

with the effective phonon self-interaction constant (nonlinearity constant)

$$
\kappa=\frac{g^{4}}{\Omega \Delta^{2}} .
$$

Here, $\rho_{z}=|+\rangle\langle+|-|-\rangle\langle-|$ with the dressed qubit states $| \pm\rangle=(|g\rangle \pm|e\rangle) / \sqrt{2}$. Therefore, if the dressed charge qubit, which was theoretically proposed [56] and has been experimentally realized [57, 58], is always in its ground state $|-\rangle$, the effective Hamiltonian for the driven NAMR is

$H_{\mathrm{eff}}=\hbar\left(\omega-\frac{g^{2}}{\Delta}\right) a^{\dagger} a+\hbar \kappa\left(a^{\dagger} a\right)^{2}+\hbar \epsilon\left(a^{\dagger} \mathrm{e}^{-i \omega_{2} t}+a \mathrm{e}^{i \omega_{2} t}\right)$.

The nonlinear Hamiltonian of the driven NAMR in Eq. (9) can also be directly obtained when the driving field is strong; however, here we only consider a weak probe current. Thus, the coupling of the NAMR to a controllable superconducting two-level is necessary for inducing phonon-phonon interactions.

\section{QUANTUM BEHAVIOR DESCRIBED BY QUASIPROBABILITY DISTRIBUTIONS}

Decoherence imposes strict conditions to observe quantum behavior in a NAMR. To demonstrate effects of the environmental on the NAMR, let us now assume that the NAMR is coupled to the thermal reservoir. Under the Markov approximation, the evolution of the reduced density operator $\rho$ for the NAMR can be described by the master equation [59]:

$$
\begin{aligned}
\frac{\partial}{\partial t} \rho= & -\frac{i}{\hbar}\left[H_{\mathrm{eff}}, \rho\right]+\frac{\gamma}{2} \bar{n}\left(2 a^{\dagger} \rho a-a a^{\dagger} \rho-\rho a a^{\dagger}\right) \\
& +\frac{\gamma}{2}(\bar{n}+1)\left(2 a \rho a^{\dagger}-a^{\dagger} a \rho-\rho a^{\dagger} a\right) .
\end{aligned}
$$

In Eq. (10), $\gamma$ is the damping rate and $\bar{n}=\left\{\exp \left[\hbar \omega /\left(k_{B} T\right)\right]-\right.$ $1\}^{-1}$ is the mean number of thermal phonons, where $k_{B}$ is the Boltzmann constant, and $T$ is the reservoir temperature at thermal equilibrium. Eq. (10) can be solved, e.g., by applying the Monte Carlo wave function simulation [59-61] and introducing the collapse operators

$$
C_{1}=\sqrt{\gamma(\bar{n}+1)} a, \quad C_{2}=\sqrt{\gamma \bar{n}} a^{\dagger} .
$$
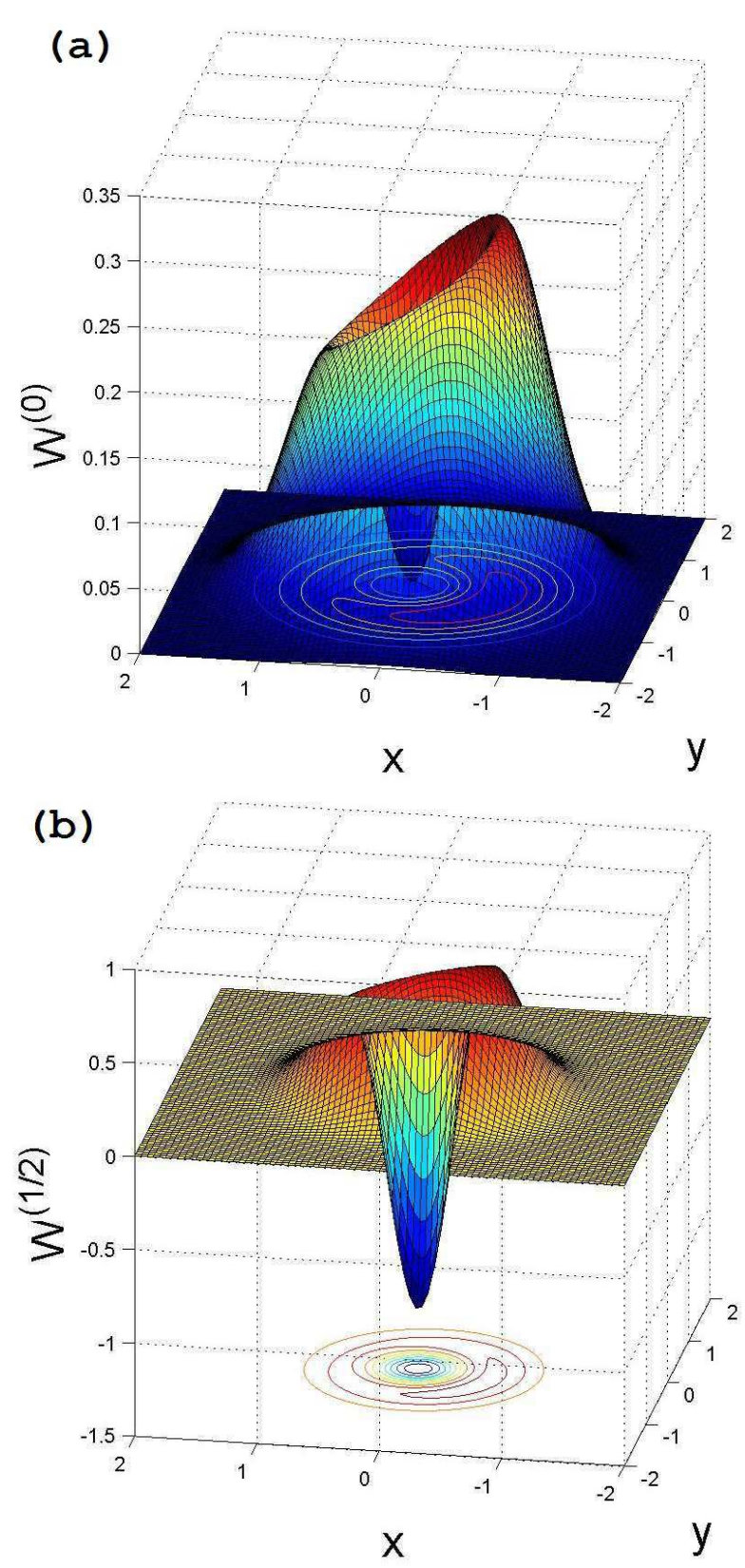

FIG. 2: (Color online) Quasidistribution functions for the NAMR steady state obtained by solving master equation (10) for $\epsilon=$ $3 \gamma, \kappa=30 \gamma$, and $\bar{n}=0.01$ with $\gamma$ as units: (a) Wigner function $W^{(0)}(x, y)$, which is non-negative in the whole phase space, and (b) 1/2-parametrized quasi-probability distribution (QPD) $W^{(1 / 2)}(x, y)$, which is negative for $\alpha=x+i y$ close to zero indicating nonclassicality of the NAMR state. The figures show the bottom of the functions.

We now study the steady-state solution, which is independent of the initial states. For the system without a drive, the time evolution ends in a state without phonons (vacuum state) at zero temperature. While for a driven system, the asymptotic state is neither the vacuum nor a pure state even at zero temperature, and can have intriguing noise properties.

A state is considered to be nonclassical if its Glauber- 
Sudarshan $P$ function cannot be interpreted as a probability density, i.e., it is negative or more singular than Dirac's $\delta$ function. Due to such singularities, it is usually hard to visualize it. To characterize the nonclassical behavior of the NAMR states generated in our system, we consider the CahillGlauber $s$-parametrized quasiprobability distribution (QPD) functions [62]:

$$
\mathcal{W}^{(s)}(\alpha)=\frac{1}{\pi} \operatorname{Tr}\left[\rho T^{(s)}(\alpha)\right],
$$

where

$$
T^{(s)}(\alpha)=\frac{1}{\pi} \int \exp \left(\alpha \xi^{*}-\alpha^{*} \xi\right) D^{(s)}(\xi) \mathrm{d}^{2} \xi,
$$

and

$$
D^{(s)}(\xi)=\exp \left(s \frac{|\xi|^{2}}{2}\right) D(\xi)
$$

with

$$
D(\xi)=\exp \left(\xi a^{\dagger}-\xi^{*} a\right)
$$

being the displacement operator. The QPD is defined for $-1 \leq s \leq 1$, which in special cases reduces to the $P$ function (for $s=1$ ), Wigner function (for $s=0$ ), and Husimi $Q$ function (for $s=-1$ ). QPDs contain the full information about states.

Let us analyze the differences between the 1/2parametrized QPD and Wigner functions under the resonant driving for the NAMR with $\omega_{2}=\omega-\left(g^{2} / \Delta\right)$. As an example, in Fig. 2, we plotted the steady-state Wigner function and 1/2-parametrized QPD, which are the numerical solutions of the master equation for a set of parameters: $\bar{n}=0.01, \epsilon=3 \gamma$, and $\kappa=30 \gamma$ in units of $\gamma$. Fig. 2 (a) shows the non-negative Wigner function of the steady state of the NAMR for these parameters. It can also be shown analytically that the steady-state Wigner function for this system is always non-negative. However, the plot for the QPD function $\mathcal{W}^{(1 / 2)}(\alpha)$ in Fig. 2(b), with the same parameters as for Fig.2(a), clearly shows negative values, corresponding to a nonclassical state of the NAMR. Below, we will discuss how to demonstrate this nonclassicality of the NAMR via the phonon blockade.

The Wigner function for the NAMR steady state is nonnegative in the whole phase space. This is in contrast to the Wigner function for various nonclassical states, including Fock states or finite superpositions of coherent states (often referred to as Schrödinger cat states) being negative in some areas of phase space. It should be noted that there are other well-known nonclassical states, including squeezed states, for which the Wigner function is non-negative as for the NAMR steady state. In general, the non-positivity of the Wigner function is a necessary but not a sufficient condition for the non-classicality. The complete characterization of the nonclassicality (the "if and only if" condition) is based on the positivity of the $P$-function. Unfortunately, this function is usually too singular to be presented graphically. The larger parameter $s$ the more nonclassical states are described by the

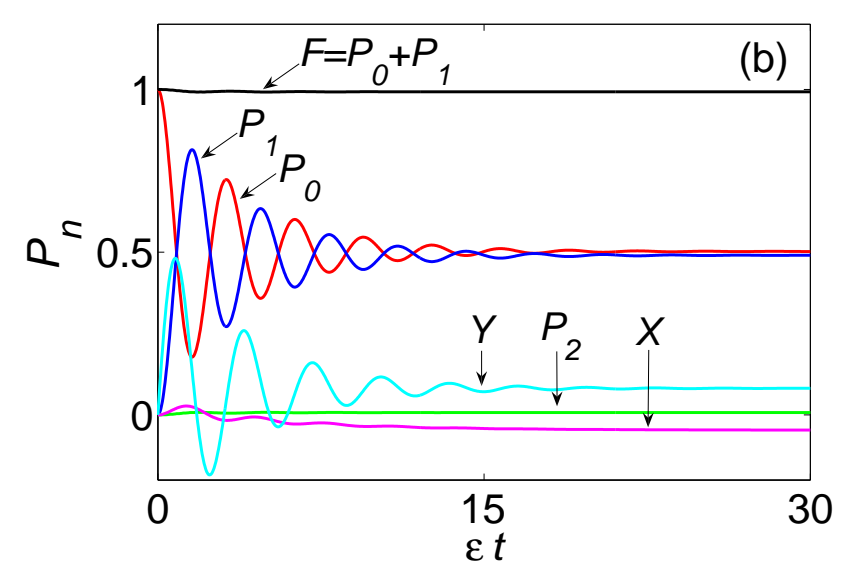

FIG. 3: (Color online) Probabilities $P_{n}=\langle n|\rho(t)| n\rangle$ of measuring $n$ phonons as a function of rescaled time, $\epsilon t$, assuming $\kappa=10 \epsilon$ and: (a) no dissipation $(\gamma=0)$ and (b) including dissipation with the same parameters as in Fig. 2. $P_{0}$ (red curves), $P_{1}$ (blue), and $P_{2}$ (green). $F=P_{0}+P_{1}$ (thick black) describes the fidelity of the phonon blockade. Additionally, the coherences $X=\operatorname{Re}\langle 0|\rho| 1\rangle$ (magenta curves) and $Y=\operatorname{Im}\langle 0|\rho| 1\rangle$ (cyan) show that the steady states partially preserve coherence.

negative $s$-parametrized QPD. In our case, to demonstrate the nonclassically of the NAMR steady state, it was enough to calculate the $s$-parametrized QPD for $s=1 / 2$ but not for $s=0$.

\section{PHONON BLOCKADE}

We now consider the case when the phonon self-interaction strength $\kappa$ is much larger than the phonon decay rate $\gamma$. When the oscillation of the NAMR is in the quantum regime, the phonon transmission can be blockaded in analogy to the single-photon blockade in a cavity [48, 49]. This is because the existence of the second phonon requires an additional en$\operatorname{ergy} \hbar \kappa$.

To demonstrate the phonon blockade, let us rewrite Eq. (9) 
as

$$
H_{\mathrm{eff}}=\hbar \bar{\omega} a^{\dagger} a+\hbar \kappa a^{\dagger} a\left(a^{\dagger} a-1\right)+\hbar \epsilon\left(a^{\dagger} \mathrm{e}^{-i \omega_{2} t}+a \mathrm{e}^{i \omega_{2} t}\right)
$$

with a renormalized frequency

$$
\bar{\omega}=\omega+\kappa-\frac{g^{2}}{\Delta} .
$$

In the rotating reference frame for $V^{\prime}=\exp \left(-i \omega_{2} a^{\dagger} a t\right)$ with $\omega_{2}=\bar{\omega}$, the Hamiltonian in Eq. (16) becomes

$$
H_{\mathrm{eff}}=\hbar \kappa a^{\dagger} a\left(a^{\dagger} a-1\right)+\hbar \epsilon\left(a^{\dagger}+a\right) .
$$

It is now easy to see that the two states $|0\rangle$ and $|1\rangle$ with zero eigenvalues are degenerate in the first term $\kappa a^{\dagger} a\left(a^{\dagger} a-1\right)$ of Eq. (18). This degeneracy plays a crucial role in the phonon blockade. Indeed, if we assume that the interaction strength $\epsilon$ is much smaller than the nonlinearity constant $\kappa$ (i.e., $\epsilon \ll \kappa$ ), then the phonon eigenstates of the Hamiltonian in Eq. (18) can become a superposition of only two states, $|0\rangle$ and $|1\rangle$, in the lowest-order approximation of the expansion in the strength $\epsilon$.

We now study the solution of the Hamiltonian in Eq. 160 under the assumption of a weak driving current, i.e., $\epsilon \ll \kappa$. Using standard perturbation theory, the state governed by the time-dependent periodic Hamiltonian in Eq. (16) with the initial condition $|\psi(t=0)\rangle=|0\rangle$ can be obtained by introducing the auxiliary operator

$$
H_{F}=H_{\mathrm{eff}}-i \frac{\partial}{\partial t}
$$

based on the Floquet theory (e.g., see Ref. [63]). The solution can be approximately given as

$$
|\psi(t)\rangle=\cos (\epsilon t)|0\rangle-i \sin (\epsilon t)|1\rangle+\mathcal{O}\left(\epsilon^{2}\right) .
$$

The solution in Eq. (20) shows that the number of phonons varies between 0 and 1 if all terms proportional to $\epsilon^{2}$ are neglected. In this small $\epsilon$ limit, the Floquet solution (20) explicitly demonstrates the phonon blockade in analogy to the photon blockade [49] or the Coulomb blockade [50], i.e., there is only one-phonon excitation and the excitation with more than one phonon is negligibly small. The photon blockade is also referred to as the optical state truncation [48, 64].

The phonon-blockaded state is nonclassical as it is a superposition of a finite number (practically two) of Fock states. Only (some) superpositions of an infinite number of Fock states can be considered classical.

The time-dependent probabilities $P_{n}=\langle n|\rho(t)| n\rangle$ of measuring the $n$-phonon state with and without dissipation are numerically simulated using the Monte Carlo method. In the ideal non-dissipative case, as shown in Fig. 3 3 a), the sum of the probabilities $P_{0}$ and $P_{1}$ with phonon numbers 0 and 1 is almost one, which means that phonon blockade occurs. For the dissipative case, Fig. 3 (b) shows the time evolutions of the elements $\langle m|\rho(t)| n\rangle$ (with $m, n=0,1$ ) for the same parameters as those in Fig. 2. The amplitudes of $P_{0}$ and $P_{1}$ exhibit decaying oscillations; however, their sum is still near one and thus the sum of other probabilities $P_{n}$ with $n>1$ is near zero.
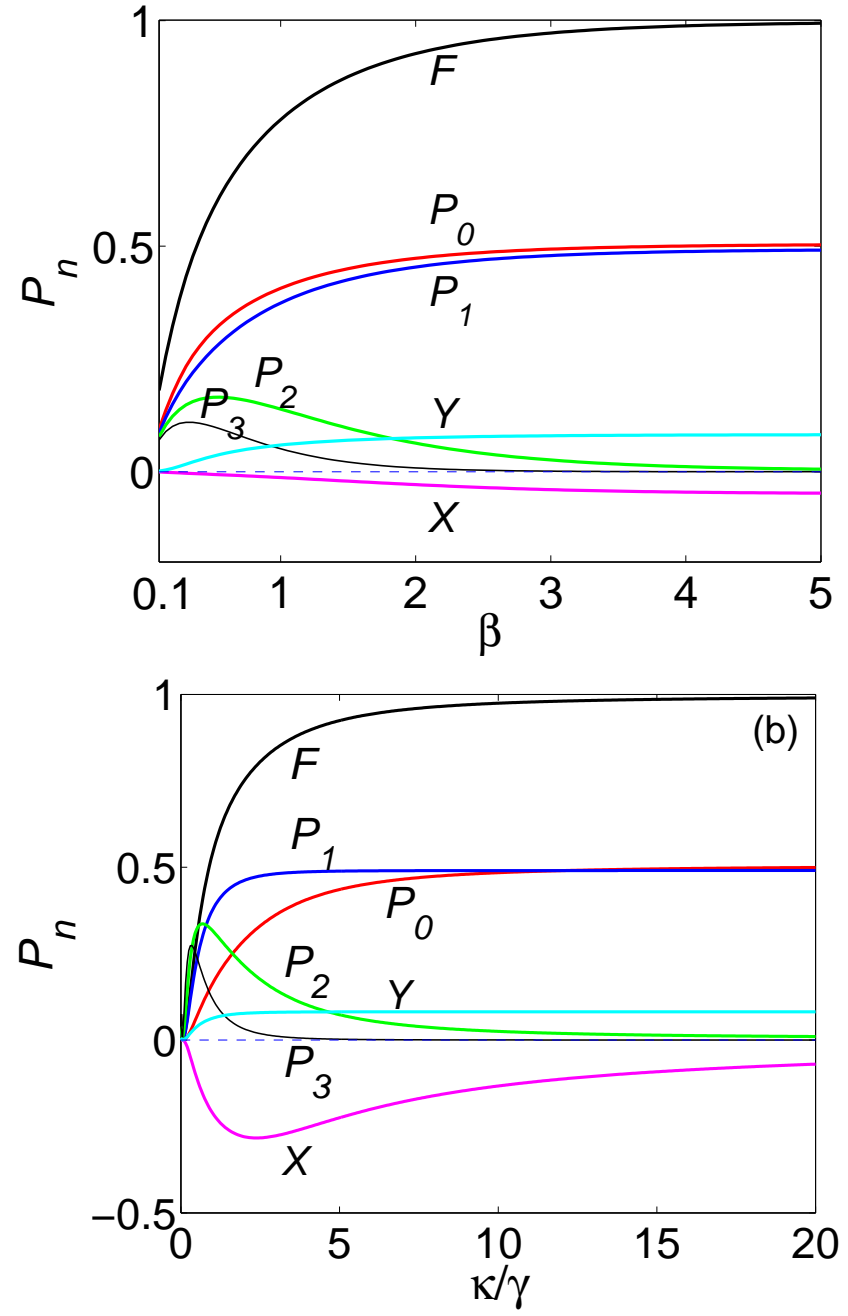

FIG. 4: (Color online) Probabilities $P_{n}$, fidelity $F$, and coherences $X$ and $Y$ for steady states as a function of (a) $\beta=\hbar \omega /\left(k_{B} T\right)$, assuming $\kappa=10 \epsilon$, and of (b) $\kappa / \gamma$, assuming $\bar{n}=0.01$, which corresponds to $\beta \approx 4.6$. In both (a) and (b), we set $\gamma=1$. The other parameters are the same as in Fig. 3 .

Therefore, the phonon blockade occurs even in the long-time limit (e.g., steady state). The non-zero off-diagonal element $\langle 0|\rho(t)| 1\rangle$ shown in Fig. 3 b) in the steady-state means that the NAMR is in the nonclassical state, which is also consistent with the steady-state plot of the QPD in Fig. 2(b). Thus, we see that the non-negative Wigner function does not directly indicate that the state is nonclassical.

To study how the environmental temperature $T$ affects the phonon blockade, the probability distributions $P_{n}=$ $\langle n|\rho(t)| n\rangle$ (for $n=0,1,2,3$ ) are plotted via the rescaled inverted temperature $\hbar \omega /\left(k_{B} T\right)$ in Fig. 4 (a). It clearly shows that the phonon blockade cannot be achieved when the thermal energy is much larger than that of the NAMR. The $\kappa$ dependent matrix elements $\langle m|\rho(t)| n\rangle$ are plotted in Fig. [4(b), which shows that the larger nonlinearity parameter $\kappa$ corresponds to a more effective phonon blockade. However, to observe the phonon blockade, it is enough to make $\kappa$ larger than 
a certain value. For instance, if the ratio between $\kappa / \gamma$ is larger than 10 , then the sum of the probabilities $P_{0}$ and $P_{1}$ is more than 0.95 , and the phonon blockade should occur.

Let us make a few comments to clarify the relation between the phonon blockade and nonclassicality in terms of the $s$ parametrized QPDs: (i) If the $s$-parametrized QPD, for some $s \in(-1,1]$ and for a given state, is negative in some region of the phase space, then the state is nonclassical. (ii) Even if the phonon blockade is not observed (for a given choice of parameters $\epsilon, \kappa, \gamma$, and $\bar{n}$ ), the $1 / 2$-parametrized QPD (or the QPD for any $s>-1$ ) can still be nonpositive. (iii) Even if we choose the parameters such that the 1/2-parametrized QPD is positive, this does not imply that the state is classical. (iv) Even if the phonon blockade does not appear, the state can be nonclassical as described by the nonpositive $P$-function (the QPD for $s=1$ ).

A good blockade of phonons can be observed for nonclassical states only. However, a poor blockade of phonons does not imply that the state is classical. Similarly to other quantum effects like squeezing or antibunching: If a specific nonclassical effect is not exhibited by a given state, it does not imply that the state is classical.

We can choose the parameters $\epsilon, \kappa, \gamma$, and $\bar{n}$ in order to observe a change (transition) from a nonpositive $1 / 2$ parametrized QPD to a positive function. However, this transition is not important in the context of nonclassicality. For various $s>-1$, one could observe such transitions for different parameters. Only the transition of the $P$-function corresponds to a transition from quantum to classical regime. As already mentioned, a good criterion of nonclassicality should be based on the $P$-function, but it is usually too singular to be presented graphically. Thus, we have chosen the QPD for another value of $s \in(0,1)$. A nonclassicality criterion based on the QPD for $s=1 / 2$ is more sensitive than that based on the Wigner function (the QPD for $s=0$ ), but still it is not sensitive enough in the general case, i.e., there are nonclassical fields described by the positive 1/2-parametrized QPD.

\section{PROPOSED MEASUREMENTS OF THE PHONON BLOCKADE}

Let us now discuss how to measure the phonon blockade via the magnetomotive technique, which is one of the basic methods to detect the motion of NAMRs [65]. As shown in Fig. 1] the induced electromotive force $V$ between two ends of the NAMR is [45, 65]

$$
V=B L \frac{p}{m}=i B L \sqrt{\frac{\hbar \omega}{2 m}}\left(a^{\dagger}-a\right),
$$

which can be experimentally measured as discussed in Ref. [65]. Here, $p$ is the momentum for the center of the NAMR mass. We analyze the power spectrum

$$
S_{V}\left(\omega^{\prime}\right)=\int_{-\infty}^{\infty}\langle V(0) V(\tau)\rangle e^{-i \omega^{\prime} t} d t
$$

defined by the Fourier transform of the induced electromotiveforce two-time correlation function

$$
\langle V(0) V(\tau)\rangle \equiv \lim _{t \rightarrow \infty}\langle V(t) V(t+\tau)\rangle
$$

This power spectrum can be measured effectively.

Power spectrum $S_{V}\left(\omega^{\prime}\right)$ and the two-time correlation function $\langle V(0) V(\tau)\rangle$ are plotted for zero temperature with different decay rates $\gamma$ in Fig. 5(a), and for a given decay rate with different temperatures $T$ (i.e., different thermal phonon number $\bar{n}$ ) in Fig. 5(b). We find that low dissipation and low temperatures produce high spectral peaks, which enable an easier observation of the phonon blockade. Thus, the environment (or some background) will limit the power spectrum for observing the phonon blockade. When $\kappa$ is negligible compared with the decay rate $\gamma$, all spectral peaks disappear and there is no phonon blockade. By other numerical calculations, we also find that a large or giant nonlinearity $\kappa$ corresponds to sharp peaks and, in this case, the phonon blockade is also easy to be observed.

Assuming perfect phonon blockade, i.e., truncation to an exact qubit state, one can analyze the whole evolution of our system confined in two-dimensional Hilbert space. To some extend this approximation can be applied in our model if the conditions $\epsilon \ll \kappa$ and $\langle\bar{n}\rangle \approx 0$ are satisfied. Then, we find that the corresponding power spectrum should have at most three peaks at frequencies

$$
\omega_{0}^{\prime}=0, \quad \omega_{1,2}^{\prime}= \pm \frac{1}{4} \sqrt{(8 \epsilon)^{2}-\gamma^{2}(1+2 \bar{n})^{2}} \approx \pm 2 \epsilon .
$$

It is seen that these frequencies are independent of $\kappa$. A peak at $\omega_{0}^{\prime}=0$ does not appear for real $\epsilon$, which is the case analyzed in the paper. Examples of such power spectra for $\omega^{\prime}>0$ are shown in Fig. 5(a) for $\bar{n}=0$ and in Fig. 5(b) for $\bar{n}=0.01$ (blue curve). In contrast, new peaks appear in the spectrum in the case of not perfect phonon blockade. This can be understood by analyzing a Hilbert space of dimension $d>2$. For example, by analyzing the system evolution confined in a three-dimensional Hilbert space, we find that the spectrum can have at most seven peaks centered at

$$
\omega^{\prime} \approx 0, \pm 2 \epsilon(1-\delta), \pm[2 \kappa(1+6 \delta) \pm \epsilon(1-\delta)]
$$

where $\delta=\epsilon^{2} /\left(8 \kappa^{2}\right)$, which depend on $\kappa$, contrary to Eq. (24). Frequencies in Eq. (25) can be approximated as $\omega^{\prime} \approx 0, \pm 2 \epsilon, \pm(2 \kappa \pm \epsilon)$. Thus, for $\omega^{\prime}>0$, the first peak occurs at $2 \epsilon$, which corresponds approximately to $\omega_{1}^{\prime}$ given in Eq. (24). The second characteristic double peak is at $2 \kappa \pm \epsilon$, as seen in Fig. 5(b) for $\bar{n}=0.5$ (red) and $\bar{n}=1$ (black curves). Eq. (25) explains only the occurrence of the first three peaks for $\omega^{\prime}>0$ in Fig. 5(b). To explain the appearance of the other two peaks at $\omega^{\prime} \approx 4 \kappa$ and $6 \kappa$, one should analyze the evolution of our system confined in (at least) four-dimensional Hilbert space. Thus, these extra peaks are a signature of a non-perfect single phonon blockade.

The spectra are not symmetric in frequency around zero, $S_{V}\left(\omega^{\prime}\right) \neq S_{V}\left(-\omega^{\prime}\right)$. Nevertheless, we depicted only the positive-frequency half of the spectra in Fig. 5 to better compare the peaks for different values of $\bar{n}$. We note that a double 
peak is observed at negative frequencies $\omega^{\prime} \approx-(2 \kappa \pm \epsilon)$ even for the cases shown in Fig. 5(a). This means that the contribution of terms $\mathcal{O}\left(\epsilon^{2}\right)$ in Eq. (20) is not negligible for the parameters chosen in Fig. 5(a), and the spectrum for $\omega^{\prime}<0$ does not correspond to a (mathematically) perfect single-phonon blockade.

In Fig. 5(b), the power spectra are plotted as a function of $\omega^{\prime} / \kappa$. There, it is seen that the position of the first positive peak depends on the ratio $\epsilon / \kappa$ in agreement with Eq. (25). The center of this peak is closer to zero for smaller ratio $\epsilon / \kappa$. However, the position of the center of the double peak (split peak) is approximately independent of $\epsilon$ and $\kappa$ (assuming that $\kappa \gg \epsilon$, so $\delta \approx 0$ ), which follows from Eq. (25). Moreover, the splitting vanishes with increasing $\gamma$. The smaller $\epsilon$ the smaller is $\gamma$ for which the splitting vanishes.

In conclusion, the observation of extra peaks at frequencies different from those in Eq. (24), show deterioration of the single-phonon blockade. The higher are such peaks the worse is the phonon blockade.

Note that the double peak at $\omega^{\prime} \approx 2 \kappa \pm \epsilon$ was found assuming the output state to be in a qutrit (three-dimensional) state. This double peak can, in general, be predicted for a qudit state, i.e., $d$-dimensional state for $2<d \ll \infty$. This corresponds to a phonon-truncation up to state $|d-1\rangle$ and can be interpreted as a generalized multi-phonon blockade. Any qudit states are nonclassical since arbitrary finite superpositions of number states are nonclassical. However, with increasing dimension $d$ of qudit states it becomes more difficult to distinguish them from classical infinite-dimensional states generated in our system. For this reason, here we analyze the standard single-phonon blockade only.

We now discuss the experimental feasibility of our proposal. With current experiments on coupling a superconducting phase [21] (or charge [23, 26, 31] or flux [46]) qubit and the NAMR, the coupling constants are on the order of hundreds of $\mathrm{MHz}$ (e.g., $200 \mathrm{MHz}$ ), the environmental temperature can reach several tens of mili-Kelvin (e.g., $20 \mathrm{mK}$ ), the frequency of the NAMR can be in the range of GHz (e.g., 1 $\mathrm{GHz}$ ). If the qubit frequency $\omega_{0}$ and the Rabi frequency $\Omega$ are taken as, e.g., $\omega_{0}=2 \mathrm{GHz}$ and $\Omega=200 \mathrm{MHz}$, then the nonlinear parameter is $\kappa=8 \mathrm{MHz}$. The observation of the phonon blockade should be possible for a quality factor $Q$ larger than $10^{3}$, which is in the NAMR quality factor range $10^{3} \sim 10^{6}$ of current experiments. By engineering $\kappa$ as in Refs. [66-68], $\kappa$ can be much larger than $8 \mathrm{MHz}$, then the phonon blockade should be easier to be observed in our proposed system.

\section{CONCLUSIONS}

We have studied the quantum mechanics of the NAMR by coupling it to a superconducting two-level system. To demonstrate our approach, a classical driving microwave is applied to the qubit so that a dressed qubit is formed. If the Rabi frequency of the driving field is strong enough, then the nonlinear phonon interaction can be induced when the dressed qubit is in its ground state. We mention that dressed charge qubits have
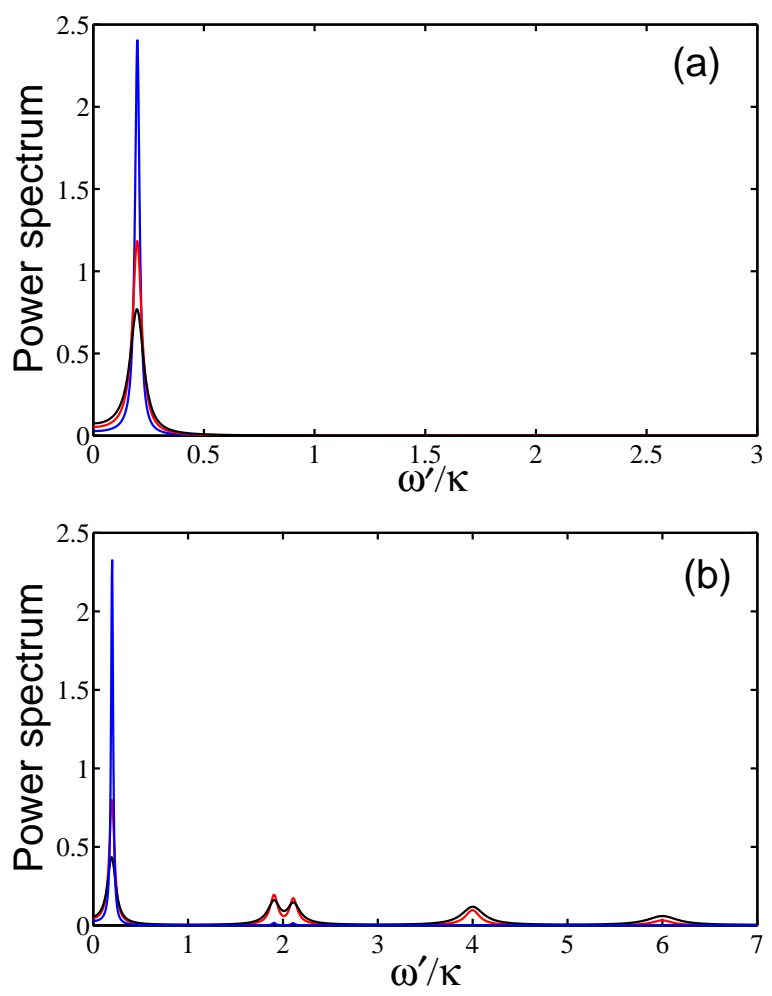

FIG. 5: (Color online) Power spectra $S_{V}\left(\omega^{\prime}\right)$ for $\kappa=30, \epsilon=3$ and: (a) $\bar{n}=0$ with $\gamma=0.5$ (blue), 1 (red), 1.5 (black), and (b) $\gamma=0.5$ with $\bar{n}=0.01$ (blue), 0.5 (red), 1 (black curves). Parameters $\kappa, \epsilon$ and $\gamma$ are in units of $g$ on the order of $\mathrm{MHz}$.

been experimentally realized [57, 58]. The dressed phase (see, e.g., Refs. [69-71]) and flux (see, e.g., Ref. [72, 73]) qubits should also be experimentally realizable.

The states of the nonlinear NAMR can be completely characterized by the Cahill-Glauber $s$-parametrized quasiprobability distribution (QPD). A state is considered to be nonclassical if it is described by a $P$-function (QPD for $s=1$ ) that cannot be interpreted as a probability density. As a drawback, the $P$-function is usually too singular to be presented graphically. Thus, other QPDs are often analyzed: If, for a given state, a QPD with $s>-1$ is negative in some regions of phasespace, then the state is nonclassical. We have shown that the Wigner function (QPD for $s=0$ ) is always non-negative for nonclassical steady states generated in our dissipative system. Thus, we have calculated the 1/2-parametrized QPD, being negative in some regions of phase-space, which clearly indicates the nonclassical character of the steady states generated in our NAMR system. Nevertheless, from an experimental point of view, the quantum-state tomography of the $1 / 2$ parametrized QPD is very challenging. Thus, we have proposed another experimentally-feasible test of nonclassicality: the phonon blockade.

We considered the case when the phonon self-interaction strength $\kappa$ significantly exceeds the phonon decay rate $\gamma$. We showed that when the NAMR oscillations are in the quantum regime, the phonon transmission can be blockaded in analogy 
to the single-photon blockade in a cavity [48, 49] or Coulomb blockade for electrons [50]. When the phonon blockade happens we also showed that a NAMR is in a nonclassical state even if its Wigner function is non-negative. Therefore, the nonclassicality of the NAMR can be demonstrated by the phonon blockade, instead of trying to detect the tiny displacements when the NAMR approaches the quantum limit. We further demonstrated that the phonon blockade can be experimentally observed by measuring the correlation spectrum of the electromotive force. All parameters in our approach are within current experimental regimes and, therefore, the quantum signature of the NAMR might be demonstrated in the near future by using this proposed approach.

We have shown that the phonon blockade can be demonstrated by a qubit-induced nonlinear NAMR. However, the temperature of the environment, the decay rate of the NAMR, the driving current, and the nonlinear coupling constant $\kappa$ limit the measured power spectrum. To more efficiently observe the phonon blockade, the following conditions should also be satisfied: (i) the temperature should be low enough so that thermal excitations should be negligibly small or the thermal energy is smaller than that of the oscillating energy of the NAMR; (ii) the quality factor of the NAMR should be high; (iii) the driving current through the NAMR should be very weak, so that the heating effect induced by the driving current can be neglected; (iv) the giant nonlinear constant $\kappa$ of the NAMR might be more useful for the phonon blockade, and this might be obtained using the approaches explored, e.g., in Refs. [66-68]. In our proposal, the larger coupling constant $g$ between the qubit and the NAMR corresponds to a larger $\kappa$, and the phonon blockade should be more easily observable for larger $\kappa$. Also the frequency of the NAMR should be large enough, so that the qubit and the NAMR are in the large detuning regime, but the detuning should not be extremely large.

\section{Acknowledgments}

FN acknowledges partial support from the Laboratory of Physical Sciences, National Security Agency, Army Research Office, National Science Foundation grant No. 0726909, JSPS-RFBR contract No. 09-02-92114, Grant-in-Aid for Scientific Research (S), MEXT Kakenhi on Quantum Cybernetics, and Funding Program for Innovative R\&D on S\&T (FIRST). YXL is supported by the National Natural Science Foundation of China under Nos. 10975080 and 60836001. YBG is supported by the NSFC Grant Nos. 10547101 and 10604002. CPS is supported by the NSFC Grant No. 10935010. AM acknowledges support from the Polish Ministry of Science and Higher Education under Grant No. 2619/B/H03/2010/38. JB was supported by the Czech Ministry of Education under Project No. MSM6198959213.
[1] X. M. H. Huang, C. A. Zorman, M. Mehregany, and M. L. Roukes, Nature (London) 421, 496 (2003).

[2] R. G. Knobel and A. N. Cleland, Nature (London) 424, 291 (2003).

[3] M. P. Blencowe, Science 304, 56 (2004).

[4] M. D. LaHaye, O. Buu, B. Camarota, and K. C. Schwab, Science 304, 74 (2004).

[5] M. P. Blencowe, Phys. Rep. 395, 159 (2004).

[6] K. C. Schwab and M. L. Roukes, Phys. Today 58 (7), 36, (2005).

[7] K. L. Ekinci and M. L. Roukes, Rev. Sc. Inst. 76, 061101 (2005).

[8] T. Carmon, H. Rokhsari, L. Yang, T. J. Kippenberg, and K. J. Vahala, Phys. Rev. Lett. 94, 223902 (2005).

[9] O. Arcizet, P. F. Cohadon, T. Briant, M. Pinard, and A. Heidmann, Nature 444, 71 (2006).

[10] S. Gigan, H. R. Bohm, M. Paternostro, F. Blaser, G. Langer, J. B. Hertzberg, K. C. Schwab, D. Bauerle, M. Aspelmeyer, and A. Zeilinger, Nature (London) 444, 67 (2006).

[11] D. Kleckner and D. Bouwmeester, Nature (London) 444, 75 (2006).

[12] G. Anetsberger, O. Arcizet, Q. P. Unterreithmeier, R. Riviere, A. Schliesser, E. M.Weig, J. P. Kotthaus, and T. J. Kippenberg, Nature Phys. 5, 909 (2009).

[13] Y. S. Park and H. Wang, Nat. Phys. 5, 489 (2009).

[14] T. J. Kippenberg and K. J. Vahala, Science 321, 1172 (2008), and references therein.

[15] C. M. Caves, K. S. Thorne, R. W. P. Drever, V. D. Sandberg, and M. Zimmermann, Rev. Mod. Phys. 52, 341 (1980).

[16] M. F. Bocko and R. Onofrio, Rev. Mod. Phys. 68, 755 (1996)
[17] E. Buks and B. Yurke, Phys. Rev. E 74, 046619 (2006).

[18] V. B. Braginsky and F. Ya. Khalili, Quantum Measurements (Cambridge University Press, Cambridge, 1992).

[19] A.D. OConnell, M. Hofheinz, M. Ansmann, R.C. Bialczak, M. Lenander, E. Lucero, M. Neeley, D. Sank, H. Wang, M. Weides, J. Wenner, J.M. Martinis, and A. N. Cleland, Nature (London) 464, 697 (2010).

[20] A. N. Cleland, Foundations of Nanomechanics (Springer, Berlin, 2003).

[21] A. N. Cleland and M. R. Geller, Phys. Rev. Lett. 93, 070501 (2004).

[22] A. D. Armour, M. P. Blencowe, and K. C. Schwab, Phys. Rev. Lett. 88, 148301 (2002).

[23] L. Tian, Phys. Rev. B 72, 195411 (2005).

[24] X. Hu and F. Nori, Phys. Rev. Lett. 76, 2294 (1996); Phys. Rev. B 53, 2419 (1996).

[25] Y. D. Wang, Y. B. Gao, and C. P. Sun, Eur. J. Phys B 40, 321 (2004).

[26] P. Rabl, A. Shnirman, and P. Zoller, Phys. Rev. B 70, 205304 (2004).

[27] F. Xue, Y. X. Liu, C. P. Sun, and F. Nori, Phys. Rev. B 76, 064305 (2007).

[28] D. H. Santamore, A. C. Doherty, and M. C. Cross, Phys. Rev. B 70, 144301 (2004).

[29] E. Buks, E. Arbel-Segev, S. Zaitsev, B. Abdo, and M. P. Blencowe, Europhys. Lett. 81, 10001 (2008).

[30] F. L. Semiao, K. Furuya, and G. J. Milburn, Phys. Rev. A 79, 063811 (2009).

[31] I. Martin, A. Shnirman, L. Tian, and P. Zoller, Phys. Rev. B 69, 125339 (2004). 
[32] K. Jacobs, P. Lougovski, and M. P. Blencowe, Phys. Rev. Lett. 98, 147201 (2007); K. Jacobs, ibid. 99, 117203 (2007).

[33] N. Grønbech-Jensen and M. Cirillo, Phys. Rev. Lett. 95, 067001 (2005); A. N. Omelyanchouk, S. N. Shevchenko, A. M. Zagoskin, E. Il'ichev, and F. Nori, Phys. Rev. B 78, 054512 (2008).

[34] S. N. Shevchenko, A. N. Omelyanchouk, A. M. Zagoskin, S. Savel'ev, and F. Nori, New J. Phys. 10, 073026 (2008).

[35] N. Lambert, C. Emary, Y.N. Chen, and F. Nori, e-print arXiv:1002.3020, N. Lambert, Y.N. Chen, J.R. Johansson, and F. Nori, Phys. Rev. B 80, 165308 (2009).

[36] E. K. Irish and K. C. Schwab, Phys. Rev. B 68, 155311 (2003).

[37] Z. R. Gong, H. Ian, Y. X. Liu, C. P. Sun, and F. Nori, Phys. Rev. A 80, 065801 (2009).

[38] S. Savel'ev, A.L. Rakhmanov, X. Hu, A. Kasumov, and F. Nori, Phys. Rev. B 75, 165417 (2007); S. Savel'ev, X. Hu, and F. Nori, New J. Phys. 8, 105 (2006).

[39] M. J. Woolley, G. J. Milburn, and C. M. Caves, New J. Phys. 10, 125018 (2008).

[40] A. Naik, O. Buu, M. D. LaHaye, A. D. Armour, A. A. Clerk, M. P. Blencowe, and K. C. Schwab, Nature (London) 443, 193 (2006).

[41] L. F. Wei, Y. X. Liu, C. P. Sun, and F. Nori, Phys. Rev. Lett. 97, 237201 (2006).

[42] K. Srinivasan and O. Painter, Nature (London) 450, 862 (2007).

[43] C. A. Regal, J. D. Teufel, and K. W. Lehnert, Nat. Phys. 4, 555 (2008).

[44] N. Lambert and F. Nori, Phys. Rev. B 78, 214302 (2008).

[45] Y. B. Gao, S. Yang, Y. X. Liu, C. P. Sun, and F. Nori, e-print arXiv:0902.2512.

[46] S. Etaki, M. Poot, I. Mahboob, K. Onomitsu, H. Yamaguchi, and H. S. J. van der Zant, Nat. Phys. 4, 785 (2008).

[47] T. F. Li, Yu. A. Pashkin, O. Astafiev, Y. Nakamura, J. S. Tsai, and H. Im, Appl. Phys. Lett. 91, 033107 (2007).

[48] W. Leoński and R. Tanaś, Phys. Rev. A 49, R20 (1994); W. Leoński, ibid. 55, 3874 (1997); A. Miranowicz, W. Leoński, S. Dyrting, and R. Tanaś, Acta Phys. Slov. 46, 451 (1996); W. Leoński, S. Dyrting, and R. Tanaś, J. Mod. Opt. 44, 2105 (1997).

[49] A. Imamoğlu, H. Schmidt, G. Woods, and M. Deutsch, Phys. Rev. Lett. 79, 1467 (1998).

[50] M. A. Kastner, Physics Today 46 (1), 24 (1993).

[51] Y. Makhlim, G. Schön, and A. Shnirman, Rev. Mod. Phys. 73, 357 (2001).

[52] J. Q. You and F. Nori, Phys. Today 58 (11), 42 (2005).
[53] J. Clarke and F. K. Wilhelm, Nature (London) 453, 1031 (2008).

[54] G. Wendin and V. S. Shumeiko, in: Handbook of Theoretical and Computational Nanotechnology, edited by M. Rieth and W. Schommers (ASP, Los Angeles, 2006).

[55] D. Zueco, G. M. Reuther, S. Kohler, and P. Hanggi, Phys. Rev. A 80, 033846 (2009).

[56] Y. X. Liu, C. P. Sun, and F. Nori, Phys. Rev. A 74, 052321 (2006).

[57] C. M. Wilson, T. Duty, F. Persson, M. Sandberg, G. Johansson, and P. Delsing, Phys. Rev. Lett. 98, 257003 (2007); J. R. Johansson, G. Johansson, C. M. Wilson, and F. Nori, Phys. Rev. Lett. 103, 147003 (2009).

[58] C. M. Wilson, G. Johansson, T. Duty, F. Persson, M. Sandberg, and P. Delsing, Phys. Rev. B 81, 024520 (2010).

[59] H. Carmichael, Statistical Methods in Quantum Optics (Springer, Berlin, 1999).

[60] J. Dalibard, Y. Castin, and K. Mølmer, Phys. Rev. Lett. 68, 580 (1992).

[61] S. M. Tan, J. Opt. B: Quantum Semiclass. Opt. 1, 424 (1999).

[62] K. E. Cahill and R. J. Glauber, Phys. Rev. 177, 1857 (1969).

[63] U. Peskin and N. Moiseyev, J. Chem. Phys. 99, 4590 (1993).

[64] W. Leoński and A. Miranowicz, Adv. Chem. Phys. 119, 195 (2001); A. Miranowicz, W. Leoński, and N. Imoto, ibid. 119, 155 (2001).

[65] K. L. Ekinci, Small 1, 786 (2005).

[66] K. Jacobs and A. J. Landahl, Phys. Rev. Lett. 103, 067201 (2009).

[67] K. M. Gheri, W. Alge, and P. Grangier, Phys. Rev. A 60, R2673 (1999).

[68] S. Rebić, J. Twamley, and G. J. Milburn, Phys. Rev. Lett. 103, 150503 (2009).

[69] L. F. Wei, Y. X. Liu, F. Nori, Europhys. Lett. 67, 1004 (2004).

[70] M. Hofheinz, E. M. Weig, M. Ansmann, R. C. Bialczak, E. Lucero, M. Neeley, A. D. O'Connell, H. Wang, J. M. Martinis, and A. N. Cleland, Nature (London) 454, 310 (2008).

[71] M. Hofheinz, H. Wang, M. Ansmann, R. C. Bialczak, E. Lucero, M. Neeley, A. D. O'Connell, D. Sank, J. Wenner, J. M. Martinis, and A. N. Cleland, Nature (London) 459, 546 (2009).

[72] Y. X. Liu, J. Q. You, L. F. Wei, C. P. Sun, and F. Nori, Phys. Rev. Lett. 95, 087001 (2005).

[73] F. Deppe, M. Mariantoni, E. P. Menzel, A. Marx, S. Saito, K. Kakuyanagi, H. Tanaka, T. Meno, K. Semba, H. Takayanagi, E. Solano, and R. Gross, Nat. Phys. 4, 686 (2008). 


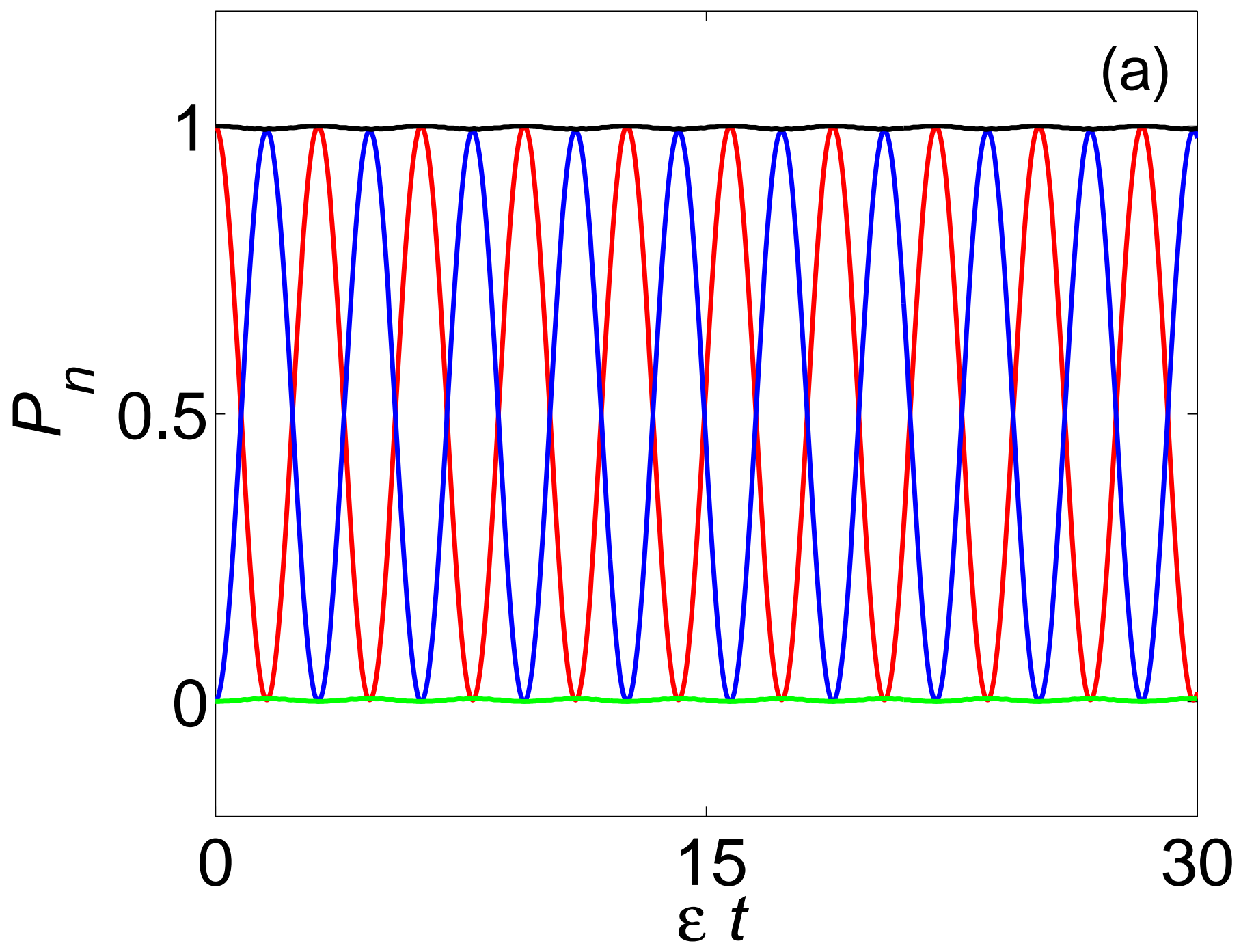

